\title{
Robust Strictly Positive Real Synthesis for Polynomial Families of Arbitrary Order ${ }^{1}$
}

\author{
WenshengYu LongWang \\ Center for Systems and Control \\ Department of Mechanics and Engineering Science, Peking University \\ Beijing 100871, P. R. China, E-mail: longwang@mech.pku.edu.cn
}

\begin{abstract}
For any two $n$-th order polynomials $a(s)$ and $b(s)$, the Hurwitz stability of their convex combination is necessary and sufficient for the existence of a polynomial $c(s)$ such that $c(s) / a(s)$ and $c(s) / b(s)$ are both strictly positive real.

Keywords Robust Stability, Strict Positive Realness, Robust Analysis and Synthesis, Polynomial Segment
\end{abstract}

\section{Introduction}

The strict positive realness (SPR) of transfer functions is an important performance specification, and plays a critical role in various fields such as absolute stability/hyperstability theory $[15,20]$, passivity analysis [11], quadratic optimal control [3] and adaptive system theory [16]. In recent years, stimulated by the parametrization method in robust stability analysis $[1,4,6]$, the study of robust strictly positive real systems has received much attention, and great progress has beem made $[2,5][7]$ [10] [12]-[14] [17]-[19] [21]-[35]. However, most results belong to the category of robust SPR analysis. Valuable results in robust SPR synthesis are rare. The following fundamental problem is still open $[14,18,22,23][25]-[28][30][31]-[34]$ :

Suppose $a(s)$ and $b(s)$ are two $n$-th order Hurwitz polynomials, does there exist, and how to find a (fixed) polynomial $c(s)$ such that $c(s) / a(s)$ and $c(s) / b(s)$ are both $S P R$ ?

By the definition of SPR, it is easy to know that the Hurwitz stability of the convex combination of $a(s)$ and $b(s)$ is necessary for the existence of polynomial $c(s)$ such that $c(s) / a(s)$ and $c(s) / b(s)$ are both SPR. In $[13,14,19]$, it was proved that, if $a(s)$ and $b(s)$ have the same even (or odd) parts, such a polynomial $c(s)$ always exists; In $[2,13,14,17,22,23,25,27,30,31]$, it was proved that, if $n \leq 4$ and $a(s), b(s) \in K$ ( $K$ is a stable interval polynomial set), such a polynomial $c(s)$ always exists; Recent results show that [22, 23, 25,27,28,30] [32]-[34], if $n \leq 6$ and $a(s)$ and $b(s)$ are the two endpoints of the convex combination of stable polynomials, such a polynomial $c(s)$ always exists. Some sufficient condition for robust SPR synthesis are presented in $[2,5,10,17,22,23,27]$, especially, the design method in $[22,23]$ is numerically efficient for high-order polynomial segments or interval polynomials, and the derived conditions are necessary and sufficient for low-order polynomial segments or interval polynomials.

This paper presents a constructive proof of the following statement: for any two $n$-th order polynomials $a(s)$ and $b(s)$, the Hurwitz stability of their convex combination is necessary and sufficient for the existence of a polynomial $c(s)$ such that $c(s) / a(s)$ and $c(s) / b(s)$ are both SPR. This also shows that the conditions given in $[22,23]$ are also necessary and sufficient and the above open problem

\footnotetext{
${ }^{1}$ Supported by National Natural Science Foundation of China (No. 60204006 and No. 69925307).
} 
admits a positive answer. Some previously obtained SPR synthesis results for low-order polynomial segments [22, 23, 25, 27, 28, 30] [32]-[34] become special cases of our main result in this paper.

\section{Main Results}

In this paper, $P^{n}$ stands for the set of $n$-th order polynomials of $s$ with real coefficients, $R$ stands for the field of real numbers, $\partial(p)$ stands for the order of polynomial $p(\cdot)$, and $H^{n} \subset P^{n}$ stands for the set of $n$-th order Hurwitz stable polynomials with real coefficients.

In the following definition, $p(\cdot) \in P^{m}, q(\cdot) \in P^{n}, f(s)=p(s) / q(s)$ is a rational function.

Definition $1[29] \quad f(s)$ is said to be strictly positive real(SPR), if

(i) $\partial(p)=\partial(q)$;

(ii) $f(s)$ is analytic in $\operatorname{Re}[s] \geq 0$, (namely, $q(\cdot) \in H^{n}$ );

(iii) $\operatorname{Re}[f(j \omega)]>0, \quad \forall \omega \in R$.

If $f(s)=p(s) / q(s)$ is proper, it is easy to get the following property:

Property 1 [9] If $f(s)=p(s) / q(s)$ is a proper rational function, $q(s) \in H^{n}$, and $\forall \omega \in$ $R, \operatorname{Re}[f(j \omega)]>0$, then $p(s) \in H^{n} \cup H^{n-1}$.

The following theorem is the main result of this paper:

Theorem 1 Suppose $a(s)=s^{n}+a_{1} s^{n-1}+\cdots+a_{n} \in H^{n}, b(s)=s^{n}+b_{1} s^{n-1}+\cdots+b_{n} \in H^{n}$, the necessary and sufficient condition for the existence of a polynomial $c(s)$ such that $c(s) / a(s)$ and $c(s) / b(s)$ are both Strict Positive Real, is

$$
\lambda b(s)+(1-\lambda) a(s) \in H^{n}, \lambda \in[0,1] .
$$

The statement is obviously true for the cases when $n=1$ or $n=2$. We will prove it for the case when $n \geq 3$.

Since SPR transfer functions enjoy convexity property, by Property 1, we get the necessary part of the theorem.

To prove sufficiency, we must first introduce some lemmas.

Lemma 1 Suppose $a(s)=s^{n}+a_{1} s^{n-1}+\cdots+a_{n} \in H^{n}$, then, for every $k \in\{1,2, \cdots, n-2\}$, the following quadratic curve is an ellipse in the first quadrant (i.e., $x_{i} \geq 0, i=1,2, \cdots, n-1$ ) of the $R^{n-1}$ space $\left(x_{1}, x_{2}, \cdots, x_{n-1}\right)^{2}$ :

$$
\left\{\begin{array}{l}
c_{k+1}^{2}-4 c_{k} c_{k+2}=0, \\
c_{l}=0, \\
l \in\{1,2, \cdots, n\}, l \neq k, k+1, k+2,
\end{array}\right.
$$

${ }^{2}$ When $n=3$, the ellipse equation is (see [27, 28] for details):

$$
\left(a_{2} x_{1}-a_{1} x_{2}-a_{3}\right)^{2}-4\left(a_{1}-x_{1}\right)\left(a_{3} x_{2}\right)=0 .
$$

When $n=4$, the two ellipse equations are (see [25] [30]-[32] for details):

$$
\begin{aligned}
& \left\{\begin{array}{l}
\left(a_{2} x_{1}+x_{3}-a_{1} x_{2}-a_{3}\right)^{2}-4\left(a_{1}-x_{1}\right)\left(a_{3} x_{2}-a_{2} x_{3}-a_{4} x_{1}\right)=0, \\
a_{4} x_{3}=0,
\end{array}\right. \\
& \left\{\begin{array}{l}
\left(a_{3} x_{2}-a_{2} x_{3}-a_{4} x_{1}\right)^{2}-4\left(a_{2} x_{1}+x_{3}-a_{1} x_{2}-a_{3}\right) a_{4} x_{3}=0, \\
a_{1}-x_{1}=0 .
\end{array}\right.
\end{aligned}
$$

When $n=5$, the three ellipse equations are (see [33] for details):

$$
\begin{aligned}
& \left\{\begin{array}{l}
\left(a_{2} x_{1}+x_{3}-a_{1} x_{2}-a_{3}\right)^{2}-4\left(a_{1}-x_{1}\right)\left(a_{5}+a_{3} x_{2}+a_{1} x_{4}-a_{2} x_{3}-a_{4} x_{1}\right)=0, \\
a_{4} x_{3}-a_{3} x_{4}-a_{5} x_{2}=0, a_{5} x_{4}=0,
\end{array}\right. \\
& \left\{\begin{array}{l}
\left(a_{5}+a_{3} x_{2}+a_{1} x_{4}-a_{2} x_{3}-a_{4} x_{1}\right)^{2}-4\left(a_{2} x_{1}+x_{3}-a_{1} x_{2}-a_{3}\right)\left(a_{4} x_{3}-a_{3} x_{4}-a_{5} x_{2}\right)=0, \\
a_{1}-x_{1}=0, a_{5} x_{4}=0,
\end{array}\right. \\
& \left\{\begin{array}{l}
\left(a_{4} x_{3}-a_{3} x_{4}-a_{5} x_{2}\right)^{2}-4\left(a_{5}+a_{3} x_{2}+a_{1} x_{4}-a_{2} x_{3}-a_{4} x_{1}\right) a_{5} x_{4}=0, \\
a_{1}-x_{1}=0, a_{2} x_{1}+x_{3}-a_{1} x_{2}-a_{3}=0 .
\end{array}\right.
\end{aligned}
$$


and this ellipse is tangent with the line

$$
\left\{\begin{array}{l}
c_{l}=0 \\
l \in\{1,2, \cdots, n\}, l \neq k+1, k+2,
\end{array}\right.
$$

and the line

$$
\left\{\begin{array}{l}
c_{l}=0 \\
l \in\{1,2, \cdots, n\}, l \neq k, k+1
\end{array}\right.
$$

respectively, where $c_{l}:=\sum_{j=0}^{n}(-1)^{l+j} a_{j} x_{2 l-j-1}, l=1,2, \cdots, n, a_{0}=1, x_{0}=1, a_{i}=0$ if $i<0$ or $i>n$, and $x_{i}=0$ if $i<0$ or $i>n-1$.

Proof Since $a(s)$ is Hurwitz stable, by using mathematical induction, Lemma 1 is proved by a direct calculation.

For notational simplicity, for $a(s)=s^{n}+a_{1} s^{n-1}+\cdots+a_{n} \in H^{n}, b(s)=s^{n}+b_{1} s^{n-1}+\cdots+b_{n} \in H^{n}$, $\forall k \in\{1,2, \cdots, n-2\}$, denote

$$
\begin{aligned}
\Omega_{e k}^{a}:=\left\{\left(x_{1}, x_{2}, \cdots, x_{n-1}\right) \mid\right. & c_{k+1}^{2}-4 c_{k} c_{k+2}<0, \\
& \left.c_{l}=0, l \in\{1,2, \cdots, n\}, l \neq k, k+1, k+2\right\},
\end{aligned}
$$

and

$$
\begin{array}{ll}
\Omega_{e k}^{b}:=\left\{\left(x_{1}, x_{2}, \cdots, x_{n-1}\right) \mid\right. & d_{k+1}^{2}-4 d_{k} d_{k+2}<0, \\
& \left.d_{l}=0, l \in\{1,2, \cdots, n\}, l \neq k, k+1, k+2\right\},
\end{array}
$$

where $c_{l}:=\sum_{j=0}^{n}(-1)^{l+j} a_{j} x_{2 l-j-1}, d_{l}:=\sum_{j=0}^{n}(-1)^{l+j} b_{j} x_{2 l-j-1}, l=1,2, \cdots, n, a_{0}=1, b_{0}=1, x_{0}=1$, $a_{i}=0$ and $b_{i}=0$ if $i<0$ or $i>n$, and $x_{i}=0$ if $i<0$ or $i>n-1$.

In what follows, $(A, B)$ stands for the set of points in the line segment connecting the point $A$ and the point $B$ in the $R^{n-1}$ space $\left(x_{1}, x_{2}, \cdots, x_{n-1}\right)$, not including the endpoints $A$ and $B$. Denote

$$
\begin{aligned}
\Omega^{a}:=\left\{\left(x_{1}, x_{2}, \cdots, x_{n-1}\right) \mid\right. & \left(x_{1}, x_{2}, \cdots, x_{n-1}\right) \in \bigcup_{i=1, i<j \leq n-2}^{n-3}\left(A_{i}, A_{j}\right), \\
& \left.\forall A_{i} \in \Omega_{e i}^{a}, i \in\{1,2, \cdots, n-2\}\right\}
\end{aligned}
$$

and

$$
\begin{aligned}
\Omega^{b}:=\left\{\left(x_{1}, x_{2}, \cdots, x_{n-1}\right) \mid\right. & \left(x_{1}, x_{2}, \cdots, x_{n-1}\right) \in \bigcup_{i=1, i<j \leq n-2}^{n-3}\left(B_{i}, B_{j}\right), \\
& \left.\forall B_{i} \in \Omega_{e i}^{b}, i \in\{1,2, \cdots, n-2\}\right\} .
\end{aligned}
$$

Lemma 2 Suppose $a(s)=s^{n}+a_{1} s^{n-1}+\cdots+a_{n} \in H^{n}, b(s)=s^{n}+b_{1} s^{n-1}+\cdots+b_{n} \in H^{n}$, if $\Omega^{a} \cap \Omega^{b} \neq \phi$, take $\left(x_{1}, x_{2}, \cdots, x_{n-1}\right) \in \Omega^{a} \cap \Omega^{b}$, and let $c(s):=s^{n-1}+\left(x_{1}-\varepsilon\right) s^{n-2}+x_{2} s^{n-3}+$ $\cdots+x_{n-2} s+\left(x_{n-1}+\varepsilon\right)(\varepsilon$ is a sufficiently small positive number $)$, then for $\frac{c(s)}{a(s)}$ and $\frac{c(s)}{b(s)}$, we have $\forall \omega \in R, \operatorname{Re}\left[\frac{c(j \omega)}{a(j \omega)}\right]>0$ and $\operatorname{Re}\left[\frac{c(j \omega)}{b(j \omega)}\right]>0$.

Proof Suppose $\left(x_{1}, x_{2}, \cdots, x_{n-1}\right) \in \Omega^{a} \cap \Omega^{b}$, let $c(s):=s^{n-1}+\left(x_{1}-\varepsilon\right) s^{n-2}+x_{2} s^{n-3}+\cdots+$ $x_{n-2} s+\left(x_{n-1}+\varepsilon\right), \varepsilon>0, \varepsilon$ sufficiently small.

When $n=6$, the four ellipse equations are (see [34] for details):

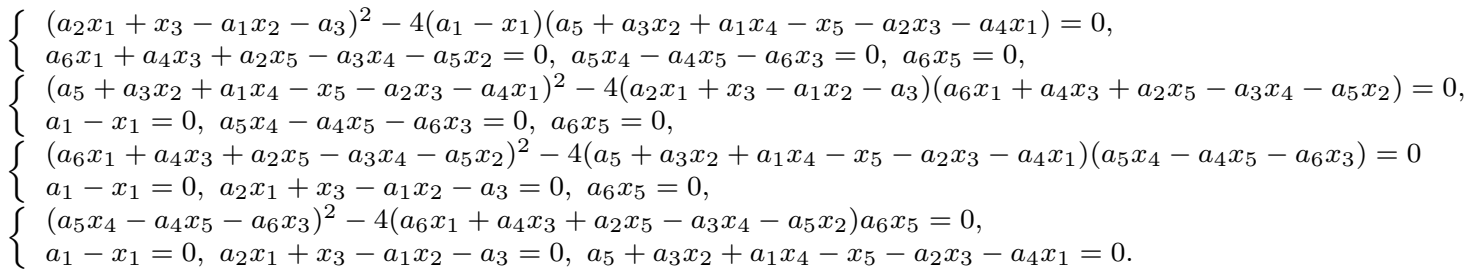


$\forall \omega \in R$, consider

$$
\begin{aligned}
\operatorname{Re}\left[\frac{c(j \omega)}{a(j \omega)}\right]= & \frac{1}{|a(j \omega)|^{2}}\left[c_{1} \omega^{2(n-1)}+c_{2} \omega^{2(n-2)}+\cdots+c_{n-1} \omega^{2}+c_{n}\right] \\
& +\operatorname{Re}\left[\frac{-\varepsilon(j \omega)^{n-2}+\varepsilon}{a(j \omega)}\right] \\
= & \frac{1}{|a(j \omega)|^{2}}\left[c_{1} \omega^{2(n-1)}+c_{2} \omega^{2(n-2)}+\cdots+c_{n-1} \omega^{2}+c_{n}\right] \\
& +\frac{(-\varepsilon)}{|a(j \omega)|^{2}}\left(-\omega^{2(n-1)}+\tilde{c}\left(\omega^{2}\right)\right),
\end{aligned}
$$

where $c_{l}:=\sum_{j=0}^{n}(-1)^{l+j} a_{j} x_{2 l-j-1}, l=1,2, \cdots, n, a_{0}=1, x_{0}=1, a_{i}=0$ if $i<0$ or $i>n$, and $x_{i}=0$ if $i<0$ or $i>n-1$, and $\tilde{c}\left(\omega^{2}\right)$ is a real polynomial with order not greater than $2(n-2)$.

In order to prove that $\forall \omega \in R, \operatorname{Re}\left[\frac{c(j \omega)}{a(j \omega)}\right]>0$, let $t=\omega^{2}$, we only need to prove that, for any $\varepsilon>0, \varepsilon$ sufficiently small, the following polynomial $f_{1}(t)$ satisfies

$$
\begin{aligned}
f_{1}(t):= & c_{1} t^{n-1}+c_{2} t^{n-2}+\cdots+c_{n-1} t+c_{n} \\
& +\varepsilon\left(t^{n-1}-\tilde{c}(t)\right)>0, \quad \forall t \in[0,+\infty) .
\end{aligned}
$$

Since $\left(x_{1}, x_{2}, \cdots, x_{n-1}\right) \in \Omega^{a}$, by the definition of $\Omega^{a}$, it is easy to know that

$$
g_{1}(t):=c_{1} t^{n-1}+c_{2} t^{n-2}+\cdots+c_{n-1} t+c_{n}>0, \quad \forall t \in(0,+\infty) .
$$

Moreover, we obviously have $f_{1}(0)>0$, and for any $\varepsilon>0$, when $t$ is a sufficiently large or sufficiently small positive number, we have $f_{1}(t)>0$, namely, there exist $0<t_{1}<t_{2}$ such that, for all $\varepsilon>0$, $t \in\left[0, t_{1}\right] \cup\left[t_{2},+\infty\right)$, we have $f_{1}(t)>0$.

Denote

$$
\begin{gathered}
M_{1}=\inf _{t \in\left[t_{1}, t_{2}\right]} g_{1}(t), \\
\left.N_{1}=\sup _{t \in\left[t_{1}, t_{2}\right]} \mid t^{n-1}-\tilde{c}(t)\right) \mid .
\end{gathered}
$$

Then $M_{1}>0$ and $N_{1}>0$. Choosing $0<\varepsilon<\frac{M_{1}}{N_{1}}$, by a direct calculation, we have

$$
\begin{aligned}
f_{1}(t):= & c_{1} t^{n-1}+c_{2} t^{n-2}+\cdots+c_{n-1} t+c_{n} \\
& +\varepsilon\left(t^{n-1}-\tilde{c}(t)\right)>0, \quad \forall t \in[0,+\infty) .
\end{aligned}
$$

Namely,

$$
\forall \omega \in R, \operatorname{Re}\left[\frac{c(j \omega)}{a(j \omega)}\right]>0 .
$$

Similarly, since $\left(x_{1}, x_{2}, \cdots, x_{n-1}\right) \in \Omega^{b}$, there exist $0<t_{3}<t_{4}$ such that, for all $\varepsilon>0, t \in$ $\left[0, t_{3}\right] \cup\left[t_{4},+\infty\right)$, we have $f_{2}(t)>0$, where

$$
\begin{aligned}
& f_{2}(t):= d_{1} t^{n-1}+d_{2} t^{n-2}+\cdots+d_{n-1} t+d_{n} \\
&+\varepsilon\left(t^{n-1}-\tilde{d}(t)\right), \\
& d_{l}:=\sum_{j=0}^{n}(-1)^{l+j} b_{j} x_{2 l-j-1}, \quad l=1,2, \cdots, n,
\end{aligned}
$$

where $b_{0}=1, x_{0}=1, b_{i}=0$ if $i<0$ or $i>n$, and $x_{i}=0$ if $i<0$ or $i>n-1$, and $\tilde{d}\left(\omega^{2}\right)$ is a real polynomial with order not greater than $2(n-2)$ which is determined by the following equation:

$$
\operatorname{Re}\left[\frac{-\varepsilon(j \omega)^{n-2}+\varepsilon}{b(j \omega)}\right]=\frac{(-\varepsilon)}{|b(j \omega)|^{2}}\left(-\omega^{2(n-1)}+\tilde{d}\left(\omega^{2}\right)\right) .
$$


Denote

$$
\begin{gathered}
g_{2}(t):=d_{1} t^{n-1}+d_{2} t^{n-2}+\cdots+d_{n-1} t+d_{n} \\
M_{2}=\inf _{t \in\left[t_{3}, t_{4}\right]} g_{2}(t) \\
\left.N_{2}=\sup _{t \in\left[t_{3}, t_{4}\right]} \mid t^{n-1}-\tilde{d}(t)\right) \mid .
\end{gathered}
$$

Then $M_{2}>0$ and $N_{2}>0$. Choosing $0<\varepsilon<\frac{M_{2}}{N_{2}}$, we have

$$
\forall \omega \in R, \operatorname{Re}\left[\frac{c(j \omega)}{b(j \omega)}\right]>0 .
$$

Thus, by choosing $0<\varepsilon<\min \left\{\frac{M_{1}}{N_{1}}, \frac{M_{2}}{N_{2}}\right\}$, Lemma 2 is proved.

Lemma 3 Suppose $a(s)=s^{n}+a_{1} s^{n-1}+\cdots+a_{n} \in H^{n}, b(s)=s^{n}+b_{1} s^{n-1}+\cdots+b_{n} \in H^{n}$, if $\lambda b(s)+(1-\lambda) a(s) \in H^{n}, \lambda \in[0,1]$, then $\Omega^{a} \cap \Omega^{b} \neq \phi$

Proof If $\forall \lambda \in[0,1], a_{\lambda}(s):=\lambda b(s)+(1-\lambda) a(s) \in H^{n}$, by Lemma 1 , for any $\lambda \in[0,1]$, $\Omega_{e k}^{a_{\lambda}}, k=1,2, \cdots, n-2$, are $n-2$ ellipses in the first quadrant of the $R^{n-1}$ space $\left(x_{1}, x_{2}, \cdots, x_{n-1}\right)$.

$\forall \lambda \in[0,1]$, denote

$$
\begin{aligned}
\Omega^{a_{\lambda}}:=\left\{\left(x_{1}, x_{2}, \cdots, x_{n-1}\right) \mid\right. & \left(x_{1}, x_{2}, \cdots, x_{n-1}\right) \in \bigcup_{i=1, i<j \leq n-2}^{n-3}\left(A_{\lambda i}, A_{\lambda j}\right), \\
& \left.\forall A_{\lambda i} \in \Omega_{e i}^{a_{\lambda}}, i \in\{1,2, \cdots, n-2\}\right\}
\end{aligned}
$$

Apparently, when $\lambda$ changes continuously from 0 to $1, \Omega^{a_{\lambda}}$ will change continuously from $\Omega^{a}$ to $\Omega^{b}$, and $\Omega_{e k}^{a_{\lambda}}$ will change continuously from $\Omega_{e k}^{a}$ to $\Omega_{e k}^{b}, k=1,2, \cdots, n-2$.

Now assume $\Omega^{a} \cap \Omega^{b}=\phi$, by the definitions of $\Omega^{a}$ and $\Omega^{b}$ and Lemma $1, \exists u_{1}>0, u_{2}>0, u_{1} \neq$ $a_{1}, u_{1} \neq b_{1}$, and $\exists \tilde{k} \in\{1,2, \cdots, n-2\}$, such that the following hyperplane $L$ in the $R^{n-1}$ space $\left(x_{1}, x_{2}, \cdots, x_{n-1}\right)$

$$
L: \quad \frac{x_{1}}{u_{1}}+\frac{x_{2}}{u_{2}}+\cdots+\frac{x_{n-1}}{u_{n-1}}=1
$$

separates $\Omega^{a}$ and $\Omega^{b}$, meanwhile, $L$ is tangent with $\Omega_{e 1}^{a}, \Omega_{e 2}^{a}, \cdots, \Omega_{e(n-2)}^{a}$ and $\Omega_{e \tilde{k}}^{b}$ simultaneously (or tangent with $\Omega_{e 1}^{b}, \Omega_{e 2}^{b}, \cdots, \Omega_{e(n-2)}^{b}$ and $\Omega_{e \tilde{k}}^{a}$ simultaneously).

Without loss of generality, suppose that $L$ is tangent with $\Omega_{e 1}^{a}, \Omega_{e 2}^{a}, \cdots, \Omega_{e(n-2)}^{a}$ and $\Omega_{e \tilde{k}}^{b}$ simultaneously.

In what follows, the notation $[x]$ stands for the largest integer that is smaller than or equal to the real number $x$, and $\langle y\rangle_{z}$ stands for the remainder of the nonnegative integer $y$ divided by the positive integer $z^{3}$.

Since $L$ is tangent with $\Omega_{e 1}^{a}, \Omega_{e 2}^{a}, \cdots, \Omega_{e(n-2)}^{a}$ and $\Omega_{e \tilde{k}}^{b}$ simultaneously, note that $a(s)$ is Hurwitz stable and $u_{1}>0, u_{1} \neq a_{1}, u_{2}>0$, using mathematical induction, by a lengthy calculation, we know that the necessary and sufficient condition for $L$ being tangent with $\Omega_{e 1}^{a}, \Omega_{e 2}^{a}, \cdots, \Omega_{e(n-2)}^{a}$ simultaneously

\footnotetext{
${ }^{3}$ For example, $[1.5]=1,[0.5]=0,[-1.5]=-2$, and $\langle 0\rangle_{2}=0,\langle 1\rangle_{2}=1,\langle 11\rangle_{3}=2$.
} 
is ${ }^{4}$

$$
\sum_{i=0}^{n}(-1)^{\left[\frac{i+1}{2}\right]} a_{i} u_{1}^{\langle i+1\rangle_{2}} u_{2}^{\left[\frac{n}{2}\right]-\left[\frac{i}{2}\right]}=0
$$

and

$$
u_{j}=(-1)^{\left[\frac{j-1}{2}\right]} u_{1}^{\langle j\rangle_{2}} u_{2}^{\left[\frac{j}{2}\right]}, \quad j=3,4, \cdots, n-1,
$$

where $a_{0}=1$.

Since $u_{j}=(-1)^{\left[\frac{j-1}{2}\right]} u_{1}^{\langle j\rangle_{2}} u_{2}^{\left[\frac{j}{2}\right]}, j=3,4, \cdots, n-1, L$ is tangent with $\Omega_{e \tilde{k}}^{b}$, by a direct calculation, we have

$$
\sum_{i=0}^{n}(-1)^{\left[\frac{i+1}{2}\right]} b_{i} u_{1}^{\langle i+1\rangle_{2}} u_{2}^{\left[\frac{n}{2}\right]-\left[\frac{i}{2}\right]}=0
$$

where $b_{0}=1$.

From (1) and (3), we obviously have $\forall \lambda \in[0,1]$,

$$
\sum_{i=0}^{n}(-1)^{\left[\frac{i+1}{2}\right]} a_{\lambda i} u_{1}^{\langle i+1\rangle_{2}} u_{2}\left[\frac{n}{2}\right]-\left[\frac{i}{2}\right]=0
$$

where $a_{\lambda i}:=a_{i}+\lambda\left(b_{i}-a_{i}\right), a_{0}=1, b_{0}=1, i=0,1,2, \cdots, n$. (4) and (2) show that $L$ is also tangent with $\Omega_{e \tilde{k}}^{a_{\lambda}}(\forall \lambda \in[0,1])$, but $L$ separates $\Omega_{e \tilde{k}}^{a}$ and $\Omega_{e \tilde{k}}^{b}$, and when $\lambda$ changes continuously from 0 to $1, \Omega_{e \tilde{k}}^{a_{\lambda}}$ will change continuously from $\Omega_{e \tilde{k}}^{a}$ to $\Omega_{e \tilde{k}}^{b}$, which is obviously impossible. This completes the proof.

Lemma 4 Suppose $a(s)=s^{n}+a_{1} s^{n-1}+\cdots+a_{n} \in H^{n}, b(s)=s^{n}+b_{1} s^{n-1}+\cdots+b_{n} \in H^{n}, c(s)=$ $s^{n-1}+x_{1} s^{n-2}+\cdots+x_{n-1}$, if $\forall \omega \in R, \operatorname{Re}\left[\frac{c(j \omega)}{a(j \omega)}\right]>0$ and $\operatorname{Re}\left[\frac{c(j \omega)}{b(j \omega)}\right]>0$, take

$$
\tilde{c}(s):=c(s)+\delta \cdot h(s), \quad \delta>0, \delta \text { sufficiently small, }
$$

(where $h(s)$ is an arbitrarily given monic $n$-th order polynomial), then $\frac{\tilde{c}(s)}{a(s)}$ and $\frac{\tilde{c}(s)}{b(s)}$ are both strictly positive real.

Proof Obviously, $\partial(\tilde{c})=\partial(a)=n$, namely, $\tilde{c}(s)$ and $a(s)$ have the same order $n$. Since $a(s) \in H^{n}$, there exists $\omega_{1}>0$ such that, for all $|\omega| \geq \omega_{1}$, we have $\operatorname{Re}\left(\frac{\tilde{c}(j \omega)}{a(j \omega)}\right)>0$.

${ }^{4}$ When $n=3$, we have (see $[27,28]$ for details):

$$
u_{1} u_{2}-a_{1} u_{2}-a_{2} u_{1}+a_{3}=0 .
$$

When $n=4$, we have (see [25] [30]-[32] for details):

$$
\left\{\begin{array}{l}
u_{1} u_{2}^{2}-a_{1} u_{2}^{2}-a_{2} u_{1} u_{2}+a_{3} u_{2}+a_{4} u_{1}=0 \\
u_{3}=-u_{1} u_{2}
\end{array}\right.
$$

When $n=5$, we have (see [33] for details):

$$
\left\{\begin{array}{l}
u_{1} u_{2}^{2}-a_{1} u_{2}^{2}-a_{2} u_{1} u_{2}+a_{3} u_{2}+a_{4} u_{1}-a_{5}=0 \\
u_{3}=-u_{1} u_{2}, u_{4}=-u_{2}^{2}
\end{array}\right.
$$

When $n=6$, we have (see [34] for details):

$$
\left\{\begin{array}{l}
u_{1} u_{2}^{3}-a_{1} u_{2}^{3}-a_{2} u_{1} u_{2}^{2}+a_{3} u_{2}^{2}+a_{4} u_{1} u_{2}-a_{5} u_{2}-a_{6} u_{1}=0 \\
u_{3}=-u_{1} u_{2}, u_{4}=-u_{2}^{2}, u_{5}=u_{1} u_{2}^{2}
\end{array}\right.
$$


Denote

$$
M_{3}=\inf _{|\omega| \leq \omega_{1}} \operatorname{Re}\left(\frac{c(j \omega)}{a(j \omega)}\right), \quad N_{3}=\sup _{|\omega| \leq \omega_{1}}\left|\operatorname{Re}\left(\frac{h(j \omega)}{a(j \omega)}\right)\right|
$$

Then $M_{3}>0$ and $N_{3}>0$. Choosing $0<\varepsilon<\frac{M_{3}}{N_{3}}$, it can be directly verified that

$$
\operatorname{Re}\left(\frac{\tilde{c}(j \omega)}{a(j \omega)}\right)>0, \forall \omega \in R
$$

Similarly, $\partial(\tilde{c})=\partial(b)=n$, and there exists $\omega_{2}>0$ such that, for all $|\omega| \geq \omega_{2}$, we have $\operatorname{Re}\left(\frac{\tilde{c}(j \omega)}{b(j \omega)}\right)>$ 0 .

Denote

$$
M_{4}=\inf _{|\omega| \leq \omega_{2}} \operatorname{Re}\left(\frac{c(j \omega)}{b(j \omega)}\right), \quad N_{4}=\sup _{|\omega| \leq \omega_{2}}\left|\operatorname{Re}\left(\frac{h(j \omega)}{b(j \omega)}\right)\right|
$$

Then $M_{4}>0$ and $N_{4}>0$. Choosing $0<\varepsilon<\frac{M_{4}}{N_{4}}$, it can be directly verified that

$$
\operatorname{Re}\left(\frac{\tilde{c}(j \omega)}{b(j \omega)}\right)>0, \forall \omega \in R
$$

Thus, by choosing $0<\varepsilon<\min \left\{\frac{M_{3}}{N_{3}}, \frac{M_{4}}{N_{4}}\right\}$, Lemma 4 is proved.

The sufficiency of Theorem 1 is now proved by simply combining Lemmas 1-4.

Remark 1 From the proof of Theorem 1, we can see that this paper not only proves the existence, but also provides a design method. In fact, based on the main idea of this paper, we have developed a geometric algorithm with order reduction for robust SPR synthesis which is very efficient for high order polynomial segments [26].

Remark 2 The method provided in this paper is constructive, and is insightful and helpful in solving more general robust SPR synthesis problems for polynomial polytopes, multilinear families, etc..

Remark 3 Our main results in this paper can also be extended to discrete-time case. In fact, by applying the bilinear transformation, we can transform the unit circle into the left half plane. Hence, Theorem 1 can be generalized to discrete-time case. Moreover, in discrete-time case, the order of the polynomial obtained by our method is bounded by the order of the given polynomial segment [27, 29].

Remark 4 If $\frac{c(s)}{a(s)}$ and $\frac{c(s)}{b(s)}$ are both SPR, it is easy to know that $\forall \lambda \in[0,1], \frac{c(s)}{\lambda a(s)+(1-\lambda) b(s)}$ is also SPR.

Remark 5 The stability of polynomial segment can be checked by many efficient methods, e.g., eigenvalue method, root locus method, value set method, etc. $[1,4,6]$.

\section{Conclusions}

We have constructively proved that, for any two $n$-th order polynomials $a(s)$ and $b(s)$, the Hurwitz stability of their convex combination is necessary and sufficient for the existence of a polynomial $c(s)$ such that $c(s) / a(s)$ and $c(s) / b(s)$ are both strictly positive real. By using similar method, we can also constructively prove the existence of SPR synthesis for low order $(n \leq 4)$ interval polynomials. Namely, when $n \leq 4$, the Hurwitz stability of the four Kharitonov vertex polynomials is necessary and sufficient for the existence of a fixed polynomial such that the ratio of this polynomial to any polynomial in the interval polynomial set is $\operatorname{SPR}$ invariant $[22,23,25,27,30,31]$. The SPR synthesis problem for high order interval polynomials is currently under investigation. 


\section{References}

[1] Ackermann, J., Bartlett, A., Kaesbauer, D., Sienel, W., and Steinhauser, R., Robust Control: Systems with Uncertain Physical Parameters. Springer-Verlag, Berlin, 1993.

[2] Anderson, B. D. O., Dasgupta, S., Khargonekar, P., Kraus, F. J., and Mansou, M., Robust strict positive realness: characterization and construction, IEEE Trans. Circuits Syst., 1990, CAS-37: 869-876.

[3] Anderson, B. D. O. and Moore, J. B., Linear Optimal Control. New York: Prentice Hall, 1970.

[4] Barmish, B. R., New Tools for Robustness of Linear Systems. New York: MacMillan Publishing Company, 1994.

[5] Betser, A. and Zeheb, E., Design of robust strictly positive real transfer functions. IEEE Trans. Circuits Syst.; Part I, 1993, CAS-40: 573-580.

[6] Bhattacharyya, S. P., Chapellat, H., and Keel, L . H ., Robust Control: The Parametric Approach. New York: Prentice Hall, 1995.

[7] Bianchini, G., Synthesis of robust strictly positive real discrete-time systems with $l_{2}$ parametric pertuebations. IEEE Trans. Circuits Syst.; Part I, 2002, CAS-49: 1221-1225.

[8] Bianchini, G., Tesi, A., and Vicino, A., Synthesis of robust strictly positive real systems with $l_{2}$ parametric uncertainty. IEEE Trans. Circuits Syst.; Part I, 2001, CAS-48: 438-450.

[9] Chapellat, H., Dahleh, M., and Bhattacharyya, S. P., On robust nonlinear stability of interval control systems. IEEE Trans. Automat. Contr., 1991, AC-36: 59-69.

[10] Dasgupta, S. and Bhagwat, A. S., Conditions for designing strictly positive real transfer functions for adaptive output error identification. IEEE Trans. Circuits Syst., 1987, CAS-34: 731-737.

[11] Desoer, C. A., Vidyasagar, M. Feedback Systems: Input-Output Properties. San Diego: Academic Press, 1975.

[12] Henrion, D., Linear matrix inequalities for robust strictly positive real design. IEEE Trans. Circuits Syst.; Part I, 2002, CAS-49: 1017-1020.

[13] Hollot, C. V., Huang, L., and Xu, Z. L., Designing strictly positive real transfer function families: A necessary and sufficient condition for low degree and structured families. Proc. of Mathematical Theory of Network and Systems, (eds. Kaashoek, M. A., Van Schuppen, J. H., Ran, A. C. M.), Boston, Basel, Berlin: Birkhäuser, 1989, 215-227.

[14] Huang, L., Hollot, C. V., Xu, Z. L., Robust analysis of strictly positive real function set. Preprints of The Second Japan-China Joint Symposium on Systems Control Theory and its Applications, 1990, 210-220.

[15] Kalman, R. E., Lyapunov functions for the problem of Lur'e in automatic control. Proc. Nat. Acad. Sci.(USA), 1963, 49: 201-205.

[16] Landau, I. D., Adaptive Control: The Model Reference Approach. New York: Marcel Dekker, 1979.

[17] Marquez, H. J. and Agathoklis, P., On the existence of robust strictly positive real rational functions. IEEE Trans. Circuits Syst.; Part I, 1998, CAS-45: 962-967. 
[18] Mosquera, C. and Perez, F., Algebraic solution to the robust SPR problem for two polynomials. Automatica, 2001, 37: 757-762.

[19] Patel, V. V. and Datta, K. B., Classification of units in $H_{\infty}$ and an alternative proof of Kharitonov's theorem. IEEE Trans. Circuits Syst.; Part I, 1997, CAS-44: 454-458.

[20] Popov, V. M., Hyperstability of Control Systems. New York: Springer-Verlag, 1973.

[21] Wang, L. and Huang, L., Finite verification of strict positive realness of interval rational functions. Chinese Science Bulletin, 1991, 36: 262-264.

[22] Wang, L. and Yu, W. S., A new approach to robust synthesis of strictly positive real transfer functions. Stability and Control: Theory and Applications, 1999, 2: 13-24.

[23] Wang, L. and Yu, W. S., Complete characterization of strictly positive real regions and robust strictly positive real synthesis method. Science in China, 2000, (E)43: 97-112.

[24] Wang, L. and Yu, W. S., On robust stability of polynomials and robust strict positive realness of transfer functions. IEEE Trans. on Circuits and Syst.; Part I, 2001, CAS-48: 127-128.

[25] Wang, L. and Yu, W. S., Robust SPR synthesis for low-order polynomial segments and interval polynomials. Proceedings of the American Control Conference (ACC 2001), Crystal Gateway Marriott, Arlington, VA, USA, 2001, 3612-3617.

[26] Xie, L. J., Wang, L., and Yu, W. S., A new geometric algorithm with order reduction for robust strictly positive real synthesis. 2002 The 41st IEEE Conference on Decision and Control (CDC 2002), Las Vegas, NV, USA, 2002.

[27] Yu, W. S., Robust Strictly Positive Real Synthesis and Robust Stability Analysis. PhD Thesis, Peking University, Beijing, 1998.

[28] Yu, W. S. and Huang, L., A necessary and sufficient conditions on robust SPR stabilization for low degree systems. Chinese Science Bulletin, 1999, 44: 517-520.

[29] Yu, W. S. and Wang, L., Some remarks on the definition of strict positive realness of transfer Functions. Proceedings of Chinese Conference on Decision and Control, Northeast University Press, Shenyang, 1999, 135-139.

[30] Yu, W. S. and Wang, L., Design of strictly positive real transfer functions. IFAC Symposium on Computer Aided Control Systems Desgin (CACSD 2000), Salford, UK, 2000.

[31] Yu, W. S. and Wang, L., Anderson's claim on fourth-order SPR synthesis is true. IEEE Trans. Circuits Syst.; Part I, 2001, CAS-48: 506-509.

[32] Yu, W. S. and Wang, L., Robust SPR synthesis for fourth-order convex combinations. Progress in natural science, 2001, 11: 461-467.

[33] Yu, W. S. and Wang, L., Robust strictly positive real synthesis for convex combination of the fifth-order polynomials. Proceedings of the IEEE Symposium on Circuits and Systems Conference (ISCAS 2001), Sydney, Austrlia, 2001, Volume 1: 739-742.

[34] Yu, W. S. and Wang, L., Robust strictly positive real synthesis for convex combination of the sixth-order polynomials. Proceedings of the American Control Conference (ACC 2003), USA, 2003 (submitted).

[35] Yu, W. S., Wang, L., and Tan, M., Complete characterization of strictly positive realness regions in coefficient space. Proceedings of the IEEE Hong Kong Symposium on Robotics and Control, Hong Kong, 1999, 259-264. 